\title{
Berührungsmedizin - ein komplementärer therapeutischer Ansatz unter besonderer Berücksichtigung der Depressionsbehandlung
}

\section{Touch Medicine - a complementary therapeutic approach exemplified by the treatment of depression}

\section{(ㄷ) (ㄱ) $\odot$}

\section{Autoren}

Bruno Müller-Oerlinghausen ${ }^{1,2}$, Michael Eggart ${ }^{3}$, Henrik Norholt ${ }^{4}$, Michael Gerlach ${ }^{5}$, Gabriele Mariell Kiebgis ${ }^{6}$, Michaela Maria Arnold ${ }^{7}$, Kerstin Uvnäs Moberg ${ }^{8}$

Institute

1 Charité - Universitätsmedizin Berlin, Berlin, Deutschland

2 Medizinische Hochschule Brandenburg Theodor Fontane, Neuruppin, Deutschland

3 Hochschule Ravensburg-Weingarten, University of Applied Sciences, Fakultät Soziale Arbeit, Gesundheit und Pflege, Weingarten, Deutschland

4 SomAffect - The Somatosensory \& Affective Neuroscience Group, Liverpool, United Kingdom

5 Dr.-Reisach-Kliniken für Psychosomatik und Psychotherapie, Hochgratklinik GmbH \& Co. KG, Oberstaufen, Deutschland

6 Praxis und Ausbildungszentrum für psychoaktive Massage, Kressbronn am Bodensee, Deutschland

7 Praxis für Naturheilkunde und Berührungsmedizin, Würzburg, Deutschland

8 Department of Animal Environment and Health, Swedish University of Agricultural Sciences, Skara, Schweden

Schlüsselwörter

Berührungsmedizin, Heilsame Berührung, Depression, Frühgeborene, Interozeption, Oxytocin, CT-Afferenzen,

Psychoaktive Massage

Key words

touch medicine, salutary touch, depression, pre-term infants, interoception, oxytocin, CT afferents, psychoactive massage therapy

online publiziert 17.12 .2021

Bibliografie

Dtsch Med Wochenschr 2022; 147: e32-e40

DOI 10.1055/a-1687-2445

ISSN 0012-0472

(c) 2022. The Author(s).

This is an open access article published by Thieme under the terms of the Creative Commons Attribution-NonDerivative-NonCommercial License, permitting copying and reproduction so long as the original work is given appropriate credit. Contents may not be used for commecial purposes, or adapted, remixed, transformed or built upon. (https://creativecommons.org/licenses/by-nc-nd/4.0/)

Georg Thieme Verlag KG, Rüdigerstraße 14,

70469 Stuttgart, Germany
Korrespondenzadresse

Prof. Dr. med. Bruno Müller-Oerlinghausen

Charité - Universitätsmedizin-Berlin, 10117 Berlin,

Deutschland

Medizinische Hochschule Brandenburg Theodor Fontane,

16816 Neuruppin, Deutschland

Büro: Bartningallee 11-13, 10557 Berlin

bruno.mueller-oerlinghausen@mhb-fontane.de

\section{ZUSAMMENFASSUNG}

Haut-zu-Haut-Berührung stellt die ursprünglichste Sinneserfahrung von Mensch und Tier dar. Ein Mangel an Berührung in der Kindheit ist mit negativen Folgen für die psychosoziale und körperliche Gesundheit verbunden. Für die Entdeckung von Rezeptoren für Temperatur und Berührung im Körper wurde 2021 der Medizin-Nobelpreis verliehen. Klinische Studien belegen den Nutzen von professionellen Berührungstechniken zur Prävention und Therapie verschiedener Erkrankungen. Der breiten Anwendung einer professionellen Berührungstherapie gilt jedoch bis heute nur ein geringes klinisches Interesse. Wir schlagen eine neue Fachdisziplin der „Berührungsmedizin“ vor und spannen nachstehend einen Bogen zwischen den Erkenntnissen moderner Berührungsforschung und der klinischen Medizin. Exemplarisch steht dabei die Behandlung der primär als Leibkrankheit konzipierten Depression im Vordergrund. Kontrollierte Studien und systematische Übersichten belegen die antidepressive, anxiolytische sowie analgetische Wirksamkeit spezieller Massagetechniken in dieser Indikation. Auch für die Neonatologie, Pädiatrie, Schmerzmedizin, Onkologie und Geriatrie konnte die Wirksamkeit heilsamer Berührung gezeigt werden. Die jeweiligen Wirkmechanismen werden auf verschiedenen Konstrukt-Ebenen diskutiert. Im Vordergrund des internationalen Forschungsinteresses stehen derzeit das Interozeptionskonzept, zum anderen endokrinologische, z. B. oxytocinerge Effekte und die Aktivierung sog. CT-Afferenzen.

\section{ABSTRACT}

Skin-to-skin-contact presents the earliest sensory experience of men and animals. Deprivation of age-relevant touch experiences during infancy results in compromised psychosocial 
and biological development. The 2021 Nobel Prize in Physiology or Medicine has been awarded for the discoveries of receptors for temperature and touch. Clinical studies have demonstrated the benefit of professional salutary touch for prevention and treatment of various illnesses. However, in the present practice of medicine the application of salutary touch does not meet adequate interest. Proposing a new medical discipline "Touch Medicine" we link the findings of modern touch research to clinical medicine. The treatment of depression which we conceive primarily as a disease afflict- ing the body will serve as an example to demonstrate the usefulness of touch therapy. Controlled studies and systematic reviews have convincingly shown antidepressive, anxiolytic and analgesic effects of salutary touch. The effectiveness and efficacy of touch therapy has also been demonstrated in many areas such as neonatology, pediatrics, oncology, and geriatrics. We discuss the underlying mechanisms on various explanatory levels including interoceptive and oxytocinergic mechanisms as well as the role of $C$ tactile afferent nerve fibers.

\section{Einleitung}

Zwischenmenschliche Berührungen (engl. social touch) stellen ein menschliches Grundbedürfnis dar, da sie Empathie, Liebe, Fürsorge, Intimität und soziale Zugehörigkeit vermitteln [1, 2]. Die markinischen Berichte über den Modus der von Jesus bewirkten Heilungen, die die vormals Kranken ins soziale Leben zurückführten, illustrieren dies in besonderer Weise [3]. Ein Mangel an zärtlicher Berührung hinterlässt psychische und physische Schäden, insbesondere bei Neugeborenen und Kindern [4, 5]. Die experimentelle und klinische Forschung zur Bedeutung und zu den potenziellen Mechanismen sowohl sozialer wie heilsamer Berührung hat in den letzten Jahrzehnten eine Vielzahl von Erkenntnissen erbracht, die für viele Bereiche der klinischen Medizin relevant, doch nach unserer Erfahrung vielen Ärztinnen und Ärzten nahezu unbekannt sind. Zwar wird die moderne Medizin immer „biologischer“, körperzentrierter, übersieht jedoch häufig die Unterscheidung zwischen belebtem Leib und objektivierbarem Körper, wie sie auch modernen Embodiment-Konzepten [6] und der Rede vom „verkörperten Bewusstsein“ als Hintergrund einer Psychiatrie als Beziehungsmedizin [7, 8] zugrunde liegt. Daher wird nachstehend versucht, einen Bogen zwischen den Erkenntnissen der modernen Berührungsforschung und der klinischen Medizin zu spannen. Unser besonderes Augenmerk gilt in diesem Kontext den potenziellen Anwendungsgebieten von heilsamer Berührung, z. B. in Form des „Affective Touch“ [9] bei psychischen und psychosomatischen Störungen, die sich nicht nur isoliert, sondern typischerweise auch in Verbindung mit körperlichen Erkrankungen zeigen bzw. sich wechselseitig bedingen - es sei exemplarisch nur an die breit gefächerte Schmerzsymptomatik oder auch die erhöhte kardiovaskuläre Morbidität und Mortalität depressiver Patienten erinnert [10]. Die Integration moderner Studienergebnisse zu beispielsweise den therapeutischen bzw. präventiven Effekten spezifischer Berührungstechniken in der Schwangerschaft [11] oder der Depression in die praktische Medizin ist ein vielversprechendes Projekt, welches spürbar internationalen Aufwind erfährt [12-14]. Freilich ist bislang offen, welche Fachdisziplin innerhalb der medizinischen Profession sich dieser Aufgabe stellen will. Auch wenn wir uns im Folgenden vor allem der Depression widmen, so ist dies nur ein Ausschnitt eines breiten Anwendungsfeldes ( $\vee$ Tab. 1), das sich von der Neonatologie bis zur Geriatrie und Hospizarbeit erstreckt [15]. Wir schlagen daher eine neue Disziplin „Berührungsmedizin“ vor, die sich die Anwendung manueller Berührungstechniken zur Prävention, Therapie oder Rehabilitation zu Nutze macht. Es sei positiv vermerkt, dass heilsame Berührung in der Kranken- und Alterspflege durchaus bereits angekommen ist. Die Berührungsmedizin schließt bereits etablierte Techniken der Physiotherapie, Osteopathie oder manuellen Medizin und auch der Körperpsychotherapie selbstverständlich nicht aus, sondern integriert sie in eine erweiterte Fachdisziplin, die insbesondere auch die psychosozialen Aspekte von Gesundheit und Krankheit berücksichtigt. Wir sehen die Berührungsmedizin nicht in einem Konkurrenzverhältnis zur sprechenden Medizin oder Psychotherapie, doch vermittelt sie über die Haut und somit den Tastsinn einen unmittelbaren Zugang zu Leibempfindungen (sog. Interozeption), die neben physiologischen Regulationsmechanismen auch Verhalten, Emotion und Kognition beeinflussen [16].

\section{Meilensteine der Berührungsforschung}

Die Haut als Mittlerin der Berührung ist das größte Sinnesorgan des Menschen und bildet sich im Rahmen der embryonalen Entwicklung aus dem Ektoderm - so wie auch das Nervensystem, woraus eine ontogenetische Verbindung zwischen Haut und Psyche plausibel wird. Der Tastsinn entwickelt sich als erster Sinn bereits sehr früh im Rahmen der embryonalen Entwicklung, ungefähr um die achte Schwangerschaftswoche. Im Gegensatz zu den anderen Sinnen wird ein kompletter Ausfall des Berührungssinns wohl nie beobachtet. Ein Mensch kann taub oder blind geboren werden ohne Tastsinn aber wäre er nicht lebensfähig [17]. Wichtige Wegmarken der Berührungsforschung werden im Folgenden nachgezeichnet $[1,18]$.

In der Mitte des zwanzigsten Jahrhunderts erschienen die ersten Publikationen zur Bedeutung von Berührung, die sich in einer Schnittfläche zwischen Medizin und Entwicklungspsychologie bewegten. René Spitz [19] erkannte die biologische Notwendigkeit der liebevollen Zuwendung an institutionalisierten Säuglingen und Kleinkindern in Heimen, die nach Trennung von der Mutter zwar mit ausreichend Nahrung und guten hygienischen Standards versorgt, aber nur wenig vom Personal berührt und gehalten wurden. In der Folge kam es bei den Säuglingen zu einer hohen Sterblichkeit und erheblichen Verhaltensstörungen. Ähnliche Folgen einer Berührungsdeprivation wurden später auch bei rumänischen Waisenkindern beschrieben [2]. Wichtige Erkenntnisse der frühen Berührungsforschung wurden durch die Experimente von Harlow gewonnen, der bei von ihren Müttern isolierten Rhesusäffchen mithilfe von künstlichen Attrappen zeigte, dass die 
- Tab. 1 Wissenschaftlich belegte Wirksamkeit professioneller Berührung (Auswahl).

\begin{tabular}{|c|c|}
\hline Fachgebiet & Berührungsmedizinische Effekte \\
\hline Onkologie & $\begin{array}{l}\text { Schmerzen } \downarrow \text {, Fatigue } \downarrow \text {, Übelkeit } \downarrow \text {, Angst } \downarrow \text {, Depression } \downarrow \text { (Cassileth \& Vickers, 2004), gesundheitsbezogene Lebensqualität } \uparrow \\
\text { (Crawford et al., 2016) }\end{array}$ \\
\hline Schmerztherapie & $\begin{array}{l}\text { Subakutes und chronisches Lumbalsyndrom } \downarrow \text {, Funktionsfähigkeit } \uparrow \text { (Brosseau et al., 2012), Nacken- und Schulterschmerzen } \downarrow \\
\text { (Kong et al., 2013), Spannungskopfschmerz } \downarrow \text { (Espí-López et al., 2016), Migräne } \downarrow \text { (Chaibi et al., 2011) }\end{array}$ \\
\hline Rheumatologie & Fibromyalgie (Schmerzen $\downarrow$, Angst $\downarrow$, Depression $\downarrow$, gesundheitsbezogene Lebensqualität $\uparrow$ ) (Yuan et al., 2015) \\
\hline Kardiologie & Essenzielle Hypertonie $\downarrow$ (Xiong et al., 2015), Herzratenvariabilität $\uparrow$ (Meier et al., 2020) \\
\hline Gastroenterologie & Obstipation $\downarrow$ (Ernst, 1999), Komplikationen bei enteraler Ernährung $\downarrow$ (Uysal et al., 2012) \\
\hline Chirurgie & Schmerzen $\downarrow$, Angst $\downarrow$, Entspannung $\uparrow$, Sedativa $\downarrow$ (Ramesh et al., 2015) \\
\hline Neurologie & $\begin{array}{l}\text { Multiple Sklerose (Fatigue } \downarrow \text {, Schmerzen } \downarrow \text {, Angst } \downarrow \text {, Depression } \downarrow \text {, Spastik } \downarrow \text { ) (Heidari et al., 2021), Parkinson (Entspannung } \downarrow \text {, } \\
\text { Stress } \downarrow \text {, Lebensqualität } \uparrow \text {, Schlafstörungen } \downarrow \text {, Schmerzen } \downarrow \text {, Fatigue } \downarrow \text {, Angst } \downarrow \text {, Depression } \downarrow \text { ) (Angelopoulou et al., 2020) }\end{array}$ \\
\hline Geburtshilfe & Geburtsschmerzen $\downarrow$, Angst $\downarrow$ (Smith et al., 2012), postpartale Depression $\downarrow$ (Müller \& Grunwald, 2021) \\
\hline Neonatologie & $\begin{array}{l}\text { Gewicht } \uparrow \text {, Hospitalisierungszeit } \downarrow \text { (Wang et al., 2013), Ikterus } \downarrow \text { (Lei et al., 2018), Stress durch medizinische Prozeduren } \downarrow \\
\text { (Roshanray et al., 2020), motorische Entwicklung } \uparrow \text { (Field, 2019) }\end{array}$ \\
\hline Pädiatrie & $\begin{array}{l}\text { Asthma (Lungenfunktion } \uparrow \text { ) (Wu et al., 2017), Angst } \downarrow \text {, Schlafstörungen } \downarrow \text {, Aufmerksamkeitsdefizit-/Hyperaktivitäts-Störung } \downarrow \text {, } \\
\text { Aggression } \downarrow \text {, posttraumatischer Stress } \downarrow \text {, atopische Dermatitis } \downarrow \text {, gastrointestinale Beschwerden } \downarrow \text { (Field, 2019) }\end{array}$ \\
\hline Psychiatrie & $\begin{array}{l}\text { Depression (auch als Komorbidität) } \downarrow \text {, Eigenschaftsangst } \downarrow \text { (Moyer et al., 2004; Baumgart et al., 2011), generalisierte Angststörung } \downarrow \\
\text { (Rapaport et al., 2016) }\end{array}$ \\
\hline Dermatologie & $\begin{array}{l}\text { Hypertrophe Narben nach Verbrennungen (Narbendicke } \downarrow \text {, Vaskularisation } \downarrow \text {, Pruritus } \downarrow \text {, Schmerzen } \downarrow \text {, Depression } \downarrow \text { ) } \\
\text { (Ault et al., 2018) }\end{array}$ \\
\hline Geriatrie & Agitation, Aggression und Depression bei Demenz $\downarrow$ (Margenfeld et al., 2019) \\
\hline
\end{tabular}

Stillung des Bedürfnisses nach Nähe und Körperkontakt wichtiger als die Stillung des Hungergefühls ist und Auswirkungen auf die sozioemotionale Entwicklung hat. Infolge dieser Experimente wurde die Bedeutung des Körperkontakts von Bowlby und dessen Mitarbeiterin Ainsworth als wesentliche Grundlage für das Bindungsverhalten des Menschen erkannt [12].

In der Folgezeit haben sich experimentelle Studien an Nagetieren mit den Auswirkungen der unterschiedlichen Art und Weise, wie die Mütter ihre Jungen ausgiebig und zärtlich berührten, beschäftigt - also mit einem Verhalten, das dem „Affective Touch“ beim Menschen ähnelt und von dem wir annehmen, dass es unmyelinisierte C-Fasern (s. u.) aktiviert [20-22]. Dieses spezifisch mütterliche Verhalten, das den Nachkommen epigenetisch zu verbesserten kognitiven Leistungen [23] wie auch zu einer positiven hormonalen Stressantwort [24-26] verhilft, überträgt sich durch nicht-genomische Mechanismen an die weibliche Nachkommenschaft und wird sichtbar, wenn diese selbst zu Muttertieren herangereift sind $[27,28]$.

Bei frühgeborenen Menschenkindern, die nicht im Inkubator, sondern mit viel Haut-zu-Haut-Kontakt („Känguru“) heranwachsen, konnten sowohl eine verbesserte physiologische Stabilität $[29,30]$ wie auch eine beschleunigte bzw. verbesserte Hirnreifung beobachtet werden [31-34]. Längsschnittbeobachtungen bestätigen die durch die frühe Berührungserfahrung insgesamt verbesserte Entwicklung [35, 36].

Auch zum Termin Neugeborene profitieren von verstärktem mütterlichen Haut-zu-Haut-Kontakt, wie neuere Studien gezeigt haben, welche die wegweisenden Forschungsergebnisse von Ainsworth et al. [37] oder Main [38, 39] voll bestätigt haben. Im
Kontext dieses Artikels sei speziell auf Untersuchungen zur Assoziation zwischen Art bzw. Intensität von frühem Körperkontakt, Bindung und dem Risiko späterer psychischer Störungen wie z. B. Depression hingewiesen [35, 40-45]. Früher Körperkontakt stellt folglich eine primärpräventive Intervention dar, die sich positiv auf die physische und psychosoziale Gesundheit auswirkt [46].

Ein bedeutender Wendepunkt der Berührungsforschung war in den vergangenen 30 Jahren die Entdeckung zuvor unbekannter nervaler Strukturen des taktilen Wahrnehmungssystems [9]. Eine strukturell und funktionell unabhängige Gruppe niederschwelliger Mechanorezeptoren stellt die von schwedischen Forschern in den 1980er-Jahren entdeckten C-taktilen (CT) Afferenzen in der behaarten Haut von Menschen und Säugetieren dar, die wie die gesamte C-Fasergruppe nicht myelinisiert sind und auf Berührungsqualitäten reagieren, die einer zärtlichen Berührung entsprechen (z. B. Streicheln, Liebkosen, Kraulen, sanftes Massieren). Unter experimentellen Bedingungen konnte nachgewiesen werden, dass CT-Afferenzen optimal bei einer Streichelgeschwindigkeit von 1-10 cm/s unter Anwendung von sanftem Druck und einem Temperaturoptimum, welches der Hauttemperatur entspricht, die stärkste Feuerungsrate zeigen [47]. Die Aktivierung der CT-Afferenzen geht auf der psychologischen Ebene mit einem Wohlgefühl einher, das sich aus wahrnehmungspsychologischer Sicht nicht einer Exterozeption, sondern der Interozeption (d. h. einem inneren, wohligen Leibgefühl, s. u.) zurechnen lässt [48]. fMRT-Befunde zeigten, dass die optimale Stimulation der CT-Afferenzen insbesondere kortikale limbische Regionen aktiviert, z. B. die posteriore Insula [49]. Die Verarbeitung von CT-assoziierten Berührungen zeigt Überlappungen mit Zentren auf, die auch an 
der Verarbeitung von Emotionen und sozialen Kognitionen beteiligt sind [47]. Neuere Arbeiten sprechen dafür, dass neben den CT-Afferenzen auch andere neuronale Strukturen als Basis für die wahrgenommenen Berührungseffekte eine wichtige Rolle spielen könnten.

Abseits der neuronalen „Bottom-up“-Prozesse sind an der Verarbeitung von Berührungsreizen auch „Top-Down“-Mechanismen beteiligt, die von hoher Relevanz sind. Insbesondere kontextuelle „Top-down“-Faktoren entscheiden über die Qualität des Berührungserlebens; so hängt z. B. die Rezeption einer Berührung maßgeblich von der berührenden Person ab („wer berührt?“), andererseits aber auch von der zugrunde liegenden Intention („warum wird berührt?") $[15,50]$.

\section{Berührungsmedizin am Beispiel der Depressionsbehandlung}

\section{Konzeptueller Ansatz}

Angesichts der hohen Zahl an depressiven Patienten werden zunehmend Stimmen laut, dass die bislang verfügbaren Therapien - seien sie medikamentös oder psychotherapeutisch - in der Summe eine keineswegs optimale Wirksamkeit besitzen [51-54]. Dies gilt auch für die derzeit stark propagierten Mindfulness-Techniken [55]. Etwa ein Drittel der Therapie-Responder klagt weiterhin über Restsymptome wie Schlafstörungen, Antriebslosigkeit etc. [56]. Auch zeigte sich in einer neuen Studie aus der Universität Witten/Herdecke, dass unter antidepressiver Medikation die körperliche Aktivierung durch emotionale Stimuli herabgesetzt ist [57]. Dies deckt sich mit Befunden aus einer fMRT-Studie, die dämpfende Effekte des Antidepressivums Escitalopram auf die Aktivität der Insula bei der Verarbeitung von Stimuli mit positiver oder negativer Valenz nahelegt [58]. Die Suche nach weiteren komplementären bzw. integrativen Therapiemöglichkeiten erscheint somit berechtigt.

Depression ist primär charakterisiert - unabhängig von ihrer jeweiligen Ausdrucksform (Starre oder Erregung) - durch die Anhedonie, d. h. das Nicht-mehr-lustvoll-spüren-Können, keine positiven Stimuli als solche mehr Perzipieren-Können; und dies betrifft nicht nur die kognitive Ebene, sondern das gesamte Sensorium. Der im Leibraum richtungsgebende Wechsel von Weitung und Engung im Sinne von Schmitz findet nicht mehr statt [59]. In der modernen, stark formalisierten psychiatrischen Diagnostik geht diese Grundsituation in einer international konsentierten Symptomen-Aufzählung fast unter. Auch bleibt bislang gänzlich unverstanden, in welcher Weise eine pharmakologische Behandlung die anhedonische Primärstörung positiv beeinflussen kann. Es ist bezeichnend für die Wertvorstellungen hochindustrialisierter Gesellschaften, dass der Besserung der Antriebslage („Patient konnte am Tag X wieder ... tun“) das primäre Interesse der Behandler und der Angehörigen gilt.

Im Folgenden soll zunächst in den Vordergrund gestellt werden, dass die Depression eine den ganzen Menschen affizierende Störung ist, die den Leib ebenso betrifft wie die affektive und kognitive Ebene. Die Starre und Enge, die sich übermächtig und bedrohlich um den depressiven Menschen legt, betrifft seine körperlichen Funktionen ebenso wie seine psychischen, was sich auch z. B. im veränderten Zeiterleben ausdrückt, wie es Fuchs unter Bezug auf u. a. von Gebsattel und Tellenbach dargestellt hat [60]. Der Soziologe Hartmut Rosa nimmt an, dass depressive Menschen „in eine gleichsam ,stillstehende“ Zeit fallen, die sich in eine zähe Masse verändert zu haben scheint.“ Jede „bedeutsame Verbindung zwischen Vergangenheit, Gegenwart und Zukunft“ (S. 101) geht dabei verloren [61]. Daraus lassen sich Konsequenzen für therapeutische Interventionen auf einer averbalen Ebene ziehen, worauf später näher eingegangen wird.

Der depressive Zustand wird von vielen körperlichen Störungen nicht nur, wie es gerne heißt, begleitet, sondern diese stellen ein Basissymptom der Depression dar, sodass auch von „somatopsychischen“ (anstatt psychosomatischen) Syndromen gesprochen werden könnte [62]. Der Leib des Depressiven - und „Leib“, gelebter/erlebter Leib, sei hier deutlich unterschieden vom anatomisch zergliederbaren Körper - ist krank. Thomas Fuchs spricht in diesem Kontext von der „Verkörperlichung“ des Leibes, d. h. die Kranken sind nicht mehr in ihrem Leib zu Hause, er wird als widerständiger Körper erfahren [7]. Vielfältige Formen von körperlichem Schmerz, Brustenge, Schwindel, Herzklopfen, Verlust von Appetit und Libido, Kälteschauer, Hitzewallungen, Magendruck, Unterkühlung und Verkrampfung der Glieder usw. haben wir immer wieder an unseren depressiven Patienten/innen beobachten können. Die Psychoanalytikerin Helga Pohl, die eine eigene „sensomotorische Körpertherapie“ entwickelt hat, sieht in diesen Dauerkontraktionen einen wichtigen pathogenetischen Aspekt und damit Ausgangspunkt therapeutischer Interventionen. Sie weist darauf hin, dass zwar das für die Depression zentrale Gefühl, das der Bedrückung, primär an der Vorderseite des Körpers, also im oberen Brustkorb lokalisiert wird, dennoch aber Angst und Depression im ganzen Körper empfunden werden, sodass es zu einer Anspannung der gesamten Beugemuskulatur kommt. Dementsprechend muss sich die Streckermuskulatur am Rücken und Nacken kompensatorisch anspannen. Zusätzlich wird durch die flache Atmung die Muskulatur unzureichend oxygeniert, sodass jede Bewegung extrem mühsam wird, und die Glieder bleiern schwer, wie es so viele Patienten verbalisieren [63]. So können wir diesen Zustand im Sinne Peter Sloterdijks auch als eine allgemeine Kontraktion, als seelisch-körperliche „Ich-Schrumpfung“ beschreiben [64]. Eine unserer Patientinnen beschrieb ihr Erleben folgendermaßen: „Ich zog mich innerlich immer mehr zusammen, bekam sogar körperliche Schmerzen von der Verkrümmung und Verkrampfung“. Eine andere kommentierte: „Die Disharmonie zwischen Kopf und Körper ... - als ginge der Körper verloren, weil der Kopf so stark beansprucht ist....".

Das subjektive Körpererleben in der Depression ist seltsamerweise viel seltener als bei schizophrenen Patienten systematisch untersucht worden. Studien, auch aus unserem eigenen Arbeitskreis, zeigen, dass Depressive ihre veränderte Leiblichkeit deutlich empfinden und negativ bewerten $[65,66]$. Eine neue phänomenologisch-qualitative Studie deutet ebenfalls darauf hin, dass Betroffene ihre leiblichen Empfindungen als Blockade, Schwere, Leere, Entfremdung oder Lähmung im Kopf bzw. im ganzen Körper umschreiben [67]. Unsere Grundannahme war und ist, dass es möglich sein sollte, über den Weg der heilsamen Berührung, die primär dem Zielorgan Haut gilt [68-71] einen averbalen 
Zugang zum depressiven Menschen zu gewinnen, sein NichtFühlen-Nicht-Spüren-Können quasi zu unterlaufen, seine Angst und Unruhe zu reduzieren, ihn wieder empfindungsfähiger und ausdrucksfähiger zu machen. Der Patient könnte so eine neue Gegenwart erleben. Als ein „Entmächtigen der Vergangenheit durch ein Ankommen im Hier und Jetzt“ beschrieb es der Psychiater und Philosoph Hinderk Emrich einst unseren konzeptuellen Ansatz [72]. Vorliegende klinische Studien unterstützen diese theoretische Erwartung, und in der Tat haben uns Patienten immer wieder präzise berichtet, dass während der Massage z. B. ihre depressiven Kognitionen zum Stillstand kamen.

\section{Empirische Studien}

In der Klinik des bekannten Schweizer Psychiaters Paul Kielholz erhielten alle depressiven Patienten Nackenmassage, basierend auf der Dissertation und experimentellen Studie von MaurerGroeli. Außerdem wurde als Indikator für einen Therapieerfolg die Wiedererwärmungszeit der Hände nach Kältebelastung gemessen [73]. Der klinische Psychologe Moyer und Mitarbeiter haben 2004 erstmals eine Metaanalyse der seinerzeit verfügbaren Publikationen zur antidepressiven und anxiolytischen Wirksamkeit von Massage vorgelegt und kamen zum Ergebnis, dass ihre Effektstärke derjenigen von Psychotherapie bei den berücksichtigten Indikationen entspricht [74]. Baumgart et al. [75] untersuchten nach strengen Kriterien ausgewählte und bis 2009 publizierte 22 RCTs an Patienten mit Depression oder Angststörungen. Es zeigte sich, dass die Validität publizierter Ergebnisse in neuerer Zeit deutlich zugenommen hat. Überwiegend ergab sich eine signifikante Überlegenheit von 30-60 Minuten dauernder Massage gegenüber den verschiedenen Kontrollbedingungen, wobei die Verschiedenheit der letzteren aus Sicht der Autoren der Durchführung einer Metaanalyse im Wege stand. In einigen Studien konnten auch Langzeitwirkungen wahrscheinlich gemacht werden, und insgesamt ergaben sich in Übereinstimmung mit Moyer et al. deutlichere Effekte bei depressiven Patienten als bei solchen mit einer Angsterkrankung.

Die Ergebnisse des ersten deutschen RCT an stationär-depressiven Patienten sowie an gesunden Probanden bewiesen eine eindeutige Überlegenheit der hierfür speziell entwickelten SlowStroke-Massage gegenüber einer Kontrollbedingung ohne Berührung und führten zur Zertifizierung dieser Technik durch den Verband für Physikalische Therapie [68, 76]. In neuester Zeit konnten Baumgart et al. eine überlegene analgetische und antidepressive Wirksamkeit der von ihnen entwickelten „psychoregulativen Massage“ bei Patienten mit psychosomatischen Rückenschmerzen eindrucksvoll belegen. Besonders bemerkenswert war die Nachhaltigkeit der Effekte über 3 Monate [77]. Auch Tiffany Field, Leiterin des Touch Research Institute in Miami (USA), hat schon frühzeitig in mehreren Studien die antidepressive und analgetische Wirkung von Massage unter Beweis stellen können [14]. Eine weitere kontrollierte Studie aus der Universität Würzburg an ambulanten depressiven Patienten ergab ebenfalls eine klare Überlegenheit der hier eingesetzten affektregulierenden Massage im Vergleich zu einem standardisierten Entspannungsverfahren [70].
Auch wenn aus methodischen Gründen derzeit noch manche Fragen offen bleiben [78] - beispielsweise nach der besten Kontrollbedingung in klinischen Studien - so ist dennoch ein Stand der empirischen berührungsmedizinischen Forschung erreicht, der eine Einführung dieser Behandlungsmöglichkeit an psychiatrischen oder psychosomatischen Kliniken unseres Erachtens rechtfertigt.

\section{Wirkungsmechanismen}

"Massage ist eine kleine Psychotherapie“, schrieb Möller schon 1994 [79]. Gewiss gibt es nicht „den“ Wirkungsmechanismus der beschriebenen antidepressiven sowie analgetischen Effekte von Massage, die übrigens auch z. B. bei Tumorpatienten mehrfach nachgewiesen werden konnten [80, 81], sondern sie müssen auf verschiedenen Erklärungsebenen, sowohl der neurophysiologischen wie der psychologischen, aber auch auf der immunologischen Ebene diskutiert werden [69]. Einige davon sind bereits oben angeklungen. Sicher spielt in diesem Zusammenhang die Aktivierung der CT-Afferenzen und ihre Verbindung zum interozeptiven System eine wichtige Rolle. Jedoch gibt es auch wichtige hormonale Wirkungsfaktoren, von denen den oxytocinergen Effekten eine besondere Bedeutung zukommt. Verschiedene Wirkungen einer psychoaktiven Massage (engl. „Affective Touch") sind mit hoher Wahrscheinlichkeit über das oxytocinerge System vermittelt; dies dürfte v. a. für die nachgewiesenen antidepressiven und analgetischen Effekte (s. o.) gelten [82, 83]. Deshalb soll im Nachstehenden auf das Oxytocin-System näher eingegangen werden.

Die zerebralen Oxytocin-Neurone haben ihren Ursprung im hypothalamischen Nucleus supraopticus und N. paraventricularis (NPV). Sie projizieren in die posteriore Hypophyse, von wo Oxytocin in die Blutzirkulation ausgeschüttet wird. Auf eine starke Stimulation hin setzen Dendrite und Kerne dieser Neurone auch Oxytocin intrazerebral frei [84]. Weiterhin projizieren spezielle oxytocinerge Neurone vom NPV ausgehend in verschiedene regulatorische Hirnareale, z. B. Locus caeruleus, periaquäduktales Höhlengrau, Raphe-Kerne etc., die wichtige autonom-regulatorische Funktionen haben [85]. Axonkollaterale der zur Hypophyse ziehenden Neurone erreichen auch Amygdala, Insula und Cortex [86, 87]. Dieses komplexe System führt durch Oxytocin-Freisetzung zu vielfältigen physiologischen Reaktionen: Im Vordergrund stehen dabei die Stimulierung prosozialen Verhaltens, die Minderung von Angst und Stressniveau, die Förderung von Ruhe und Wohlbefinden, analgetische und anti-inflammatorische Effekte, aber auch die Auslösung von regenerativen Prozessen [83].

Das Oxytocin-System wird in Teilen oder zur Gänze in verschiedenen Situationen aktiviert, z. B. ausgelöst durch unterschiedliche Arten sozialer Interaktion [82, 83, 88]. Dies kann auch unter somatosensorischer Stimulation wie etwa dem Geburtsvorgang [89] oder dem Stillen [90] beobachtet werden. Aber auch die Aktivierung kutaner Afferenzen, insbesondere durch sanftes Streicheln, stimuliert die Freisetzung von Oxytocin [91]. Der heutzutage gerne praktizierte enge Haut-zu-Haut-Kontakt zwischen Mutter und Neugeborenem, bei dem statischer Druck ausgeübt wird, resultiert in einer bemerkenswert starken Oxytocin-Freiset- 
zung. Diese Beobachtung spricht für eine wichtige Rolle zusätzlicher Rezeptoren in der Haut für die Hormonreaktion [92].

Da sanfte Hautberührung beim Menschen eine Oxytocin-Ausschüttung bewirkt, mit nachfolgender Aktivierung serotonerger Raphe-Kerne sowie dopaminerger Neurone im Striatum und Nucleus accumbens, legt auch die neurowissenschaftliche Erklärungsebene nahe, dass z. B. sanfte Massagen die Stimmung positiv beeinflussen [93, 94]. Die Aktivierung des oxytocinergen Systems dürfte hierbei eine bedeutsame Rolle spielen [70, 82, 83, 95, 96].

Eine andere Erklärungsebene beruht auf dem Konstrukt der Interozeption. Interozeptionen umfassen alle Leibempfindungen, die sich auf den physiologischen Zustand des gesamten Körpers beziehen - also auch viszerale Sensationen und Reize von Thermo-, Chemo-, Nozizeptoren sowie niederschwellige Mechanorezeptoren des Berührungssystems, die CT-Afferenzen [97]. Hierbei nimmt die Insula eine zentrale Rolle bei der Integration aller Einzelempfindungen zu einem leiblichen Selbst („material me“) ein [98]. Abseits homöostatischer Regulationsmechanismen legen neuere Befunde einen Einfluss der Interozeption auf Affekt, Kognition und Verhalten nahe [16].

Die Interozeptions-Forschung liefert somit bedeutende Beiträge zur Pathogenese affektiver Störungen, beispielsweise durch Bestätigung zentraler Annahmen älterer Emotionstheorien. So postulierte William James gegen Ende des 19. Jahrhunderts, dass spezifische viszerale Veränderungen in Reaktion auf einen Stimulus eintreten, die ihrerseits als Emotion empfunden werden [99]. Entsprechend fanden wir im Rahmen einer systematischen Analyse Hinweise, dass insbesondere Patienten mit mittelschwerer Depression schlechte Herzwahrnehmer sind - also die Zahl ihrer Herzschläge innerhalb definierter Zeitintervalle gegenüber gesunden Kontrollen signifikant unterschätzen, was auf eine gedämpfte kardiale Interozeption hinweist. Diese Fähigkeit steht in einer komplexen Beziehung zu affektiven und kognitiven Symptomen der Depression, z. B. zu affektiver Verflachung oder Entscheidungsschwierigkeiten [100]. Maladaptive Aufmerksamkeitsstile gegenüber Interozeptionen, wie bspw. die Sorge über unangenehme Leibempfindungen, vermindertes Körpervertrauen und gestörte interozeptive Selbstregulationsmechanismen, stellen charakteristische Symptome der Depression dar, deren Veränderungen prädiktiv für den Behandlungserfolg einer stationären Therapie sind [101, 102].

Wenn also die Massagetherapie die depressionstypische Anhedonie zu unterlaufen vermag (s. o.), so kann vermutet werden, dass ihre klinischen Effekte über das interozeptive System vermittelt werden $[71,103]$. Vorläufige Ergebnisse deuten darauf hin, dass interozeptive Zustände über die Haut beeinflussbar sind, z. B. eine durch sanfte Berührung vermittelte Erhöhung der Genauigkeit der Herzschlagwahrnehmung [104, 105]. Diese Befunde sind für die Behandlung affektiver Störungen von Bedeutung, da Depressionen mit einer verringerten Herzratenvariabilität einhergehen, die prädiktiv für kardiovaskuläre Ereignisse ist [106].

\section{Schlussfolgerungen}

Die Wirksamkeit der Berührungstherapie kann innerhalb eines breiten Indikationsspektrums von der Neonatologie bis zur Geria- trie und Palliativmedizin, insbesondere auch im Bereich der Psychiatrie/Psychosomatik als ausreichend belegt angesehen werden, auch wenn weitere klinische Studien mit erhöhten methodischen Anforderungen wünschenswert sind [78]. Zum anderen begegnen die zugrunde liegenden Wirkmechanismen auf verschiedenen Konstruktebenen derzeit einem sehr aktiven internationalen Forschungsinteresse, insbesondere psychologischer, leider deutlich seltener medizinischer Arbeitsgruppen. Daraus ergeben sich für uns 2 Desiderata: Wirksamkeit und Praktikabilität solcher berührungsmedizinischer Behandlungsformen sollten nunmehr in größerem Umfang in Klinik und Praxis und auch mit Unterstützung der Krankenkassen auf ihre Wirksamkeit und Praxistauglichkeit erprobt werden. Auch im Bereich der Pflege sollten die bereits vorhandenen positiven Erfahrungen zur Wirksamkeit heilsamer Berührung, etwa bei Demenz [107], erweitert und durch geeignete Studien bekräftigt werden. Zweitens erscheint uns dringlich, dass auch im deutschsprachigen Raum die Berührungsforschung an einigen entsprechend ausgestatteten wissenschaftlichen Zentren mit Patientenzugang (vergleichbar mit dem US-amerikanischen Touch Research Institute) konzentriert und durch wissenschaftliche Gesellschaften gestützt wird.

\section{KERNAUSSAGEN}

- Die gemeinsame Entwicklung von Haut und Nervensystem aus dem Ektoderm ist Grundlage der engen Beziehung von Tastsinn und Psyche.

- Der phänomenologische Zugang zum Wesen der Depression als Leibkrankheit eröffnet ein neues Verständnis der antidepressiven Wirksamkeit von professioneller Berührungstherapie.

- Auf der neurobiologischen Ebene stehen derzeit oxytocinerge Mechanismen, die Stimulation spezifischer Hautrezeptoren und ihre Assoziation zu interozeptiven Prozessen im Vordergrund des Interesses.

- Die inzwischen gut belegte Wirksamkeit professioneller Berührungstherapie in den verschiedensten medizinischen Indikationen rechtfertigt die Gründung einer neuen Fachdisziplin „Berührungsmedizin“.

\section{Interessenkonflikte}

BMOE, ME, HN, MG und KUM erklären, dass kein Interessenkonflikt besteht. GMK führt eine Privatpraxis für Körpertherapie und leitet Ausbildungsseminare für die von ihr entwickelte Massagemethode. MMA betreibt eine Privatpraxis für Berührungsmedizin.

\section{Danksagung}

Wir danken Herrn Prof. Dr. med. Dr. phil Thomas Fuchs, Universität Heidelberg, für die hilfreiche Durchsicht des Manuskripts. 


\section{Literatur}

[1] Thayer S. History and strategies of research on social touch. J Nonverbal Behav 1986; 10: 12-28. doi:10.1007/BF00987202

[2] Montagu A. Körperkontakt. Die Bedeutung der Haut für die Entwicklung des Menschen. 13. Aufl. Stuttgart: Klett-Cotta; 2015

[3] Wilkens L. „Deine Treue hat dich geheilt“. Studien über die Heilungsmacht Jesu und die apokalyptische Erwartung im Markusevangelium. Frankfurt am Main: Lang; 2011

[4] Cochrane N. Physical contact experience and depression. Acta Psychiatr Scand Suppl 1990; 357: 1-91

[5] Takeuchi MS, Miyaoka H, Tomoda A et al. The Effect of Interpersonal Touch During Childhood on Adult Attachment and Depression: A Neglected Area of Family and Developmental Psychology? Journal of Child and Family Studies 2010; 19: 109-117. doi:10.1007/s10826-009-9290-x

[6] Storch M, Cantieni B, Hüther G et al. Embodiment. Die Wechselwirkung von Körper und Psyche verstehen und nutzen. 3. Aufl. Bern: Hogrefe; 2017

[7] Fuchs T. Leib, Raum, Person. Entwurf einer phänomenologischen Anthropologie. 2. Aufl. Stuttgart, 2018

[8] Fuchs T. Randzonen der Erfahrung. Beiträge zur phänomenologischen Psychopathologie. Schriftenreihe der Gesellschaft für phänomenologische Anthropologie, Psychiatrie und Psychotherapie (DGAP). Freiburg, München: Verlag Karl Alber; 2020

[9] McGlone F, Wessberg J, Olausson H. Discriminative and affective touch: sensing and feeling. Neuron 2014; 82: 737-755. doi:10.1016/ j.neuron.2014.05.001

[10] Seligman F, Nemeroff CB. The interface of depression and cardiovascular disease: therapeutic implications. Ann N Y Acad Sci 2015; 1345: 25-35. doi: $10.1111 /$ nyas. 12738

[11] Kiebgis GM, Müller-Oerlinghausen B. Ich fühle, also bin ich. Deutsche Hebammen Zeitschrift 2017; 2: 18-21

[12] Norholt $\mathrm{H}$. Revisiting the roots of attachment: A review of the biological and psychological effects of maternal skin-to-skin contact and carrying of full-term infants. Infant Behav Dev 2020; 60: 101441. doi:10.1016/ j.infbeh.2020.101441

[13] Changaris M. Touch. The neurobiology of health, healing, and human connection. Mendocino, CA: LifeRhythm; 2015

[14] Field T. Touch therapy. Edinburgh, New York: Churchill Livingstone; 2000

[15] Müller-Oerlinghausen B, Kiebgis GM. Berührung - Warum wir sie brauchen und wie sie uns heilt. Berlin: Ullstein; 2018

[16] Khalsa SS, Adolphs R, Cameron OG et al. Interoception and Mental Health: A Roadmap. Biol Psychiatry Cogn Neurosci Neuroimaging 2018; 3: 501513. doi:10.1016/j.bpsc.2017.12.004

[17] Grunwald M. Homo hapticus. Warum wir ohne Tastsinn nicht leben können. München: Droemer; 2017

[18] Olausson H, Wessberg J, Morrison I, McGlone F, Hrsg. Affective touch and the neurophysiology of CT afferents. New York: Springer; 2016. doi:10.1007/978-1-4939-6418-5

[19] Spitz R. Hospitalism: an inquiry into the genesis of psychiatric conditions in early childhood. Psychoanal Study Child 1945; 1: 53-74

[20] Murgatroyd C, Quinn JP, Sharp HM et al. Effects of prenatal and postnatal depression, and maternal stroking, at the glucocorticoid receptor gene. Transl Psychiatry 2015; 5: e560. doi:10.1038/tp.2014.140

[21] Sharp H, Pickles A, Meaney M et al. Frequency of infant stroking reported by mothers moderates the effect of prenatal depression on infant behavioural and physiological outcomes. PLoS ONE 2012; 7: e45446. doi:10.1371/ journal.pone.0045446

[22] Walker SC, Cavieres A, Peñaloza-Sancho V et al. C-low threshold mechanoafferent targeted dynamic touch modulates stress resilience in rats exposed to chronic mild stress. Eur J Neurosci 2020. doi:10.1111/ejn.14951
[23] Liu D, Diorio ], Day JC et al. Maternal care, hippocampal synaptogenesis and cognitive development in rats. Nat Neurosci 2000; 3: 799-806. doi: $10.1038 / 77702$

[24] Meaney MJ. Maternal care, gene expression, and the transmission of individual differences in stress reactivity across generations. Annu Rev Neurosci 2001; 24: 1161-1192. doi:10.1146/annurev.neuro.24.1.1161

[25] Liu D, Diorio J, Tannenbaum B et al. Maternal care, hippocampal glucocorticoid receptors, and hypothalamic-pituitary-adrenal responses to stress. Science 1997; 277: 1659-1662. doi:10.1126/science.277.5332.1659

[26] Weaver ICG, Cervoni N, Champagne FA et al. Epigenetic programming by maternal behavior. Nat Neurosci 2004; 7: 847-854. doi:10.1038/ $\mathrm{nn} 1276$

[27] Champagne F, Meaney MJ. Like mother, like daughter: evidence for nongenomic transmission of parental behavior and stress responsivity. Prog Brain Res 2001; 133: 287-302. doi:10.1016/s0079-6123(01)33022-4

[28] Francis D, Diorio ], Liu D et al. Nongenomic transmission across generations of maternal behavior and stress responses in the rat. Science 1999; 286: 1155-1158. doi:10.1126/science.286.5442.1155

[29] Bergman NJ, Linley LL, Fawcus SR. Randomized controlled trial of skin-toskin contact from birth versus conventional incubator for physiological stabilization in 1200- to 2199-gram newborns. Acta Paediatr 2004; 93: 779-785. doi:10.1111/j.1651-2227.2004.tb03018.x

[30] Chi Luong K, Long Nguyen T, Huynh Thi DH et al. Newly born low birthweight infants stabilise better in skin-to-skin contact than when separated from their mothers: a randomised controlled trial. Acta Paediatr 2016; 105: 381-390. doi:10.1111/apa.13164

[31] Marvin MM, Gardner FC, Sarsfield KM et al. Increased Frequency of Skinto-Skin Contact Is Associated with Enhanced Vagal Tone and Improved Health Outcomes in Preterm Neonates. Am J Perinatol 2019; 36: 505510. doi:10.1055/s-0038-1669946

[32] Isler JR, Stark RI, Grieve PG et al. Integrated information in the EEG of preterm infants increases with family nurture intervention, age, and conscious state. PLoS ONE 2018; 13: e0206237. doi:10.1371/journal. pone. 0206237

[33] Welch MG, Myers MM, Grieve PG et al. Electroencephalographic activity of preterm infants is increased by Family Nurture Intervention: a randomized controlled trial in the NICU. Clin Neurophysiol 2014; 125: 675-684. doi:10.1016/j.clinph.2013.08.021

[34] Welch MG, Barone JL, Porges SW et al. Family nurture intervention in the $\mathrm{NICU}$ increases autonomic regulation in mothers and children at 4-5 years of age: Follow-up results from a randomized controlled trial. PLoS ONE 2020; 15: e0236930. doi:10.1371/journal.pone.0236930

[35] Feldman R, Rosenthal Z, Eidelman Al. Maternal-preterm skin-to-skin contact enhances child physiologic organization and cognitive control across the first 10 years of life. Biol Psychiatry 2014; 75: 56-64. doi:10.1016/j.biopsych.2013.08.012

[36] Ulmer Yaniv A, Salomon R, Waidergoren S et al. Synchronous caregiving from birth to adulthood tunes humans' social brain. Proc Natl Acad Sci U S A 2021; 118. doi:10.1073/pnas.2012900118

[37] Ainsworth MDS, Blehar MC, Waters E et al. Patterns of attachment: A psychological study of the strange situation. Hillsdale, New Jersey: Lawrence Erlbaum Associates; 1978

[38] Main M. Parental aversion to infant-initiated contact is correlated with the parent's own rejection during childhood: The effects of experience on signals of security with respect to attachment. In: Barnard KE, Brazelton T, Hrsg. Touch: The foundation of experience: Full revised and expanded proceedings of Johnson \& Johnson Pediatric Round TableX. International Universities Press, Inc; 1990: 461-495

[39] Main M, Stadtman J. Infant response to rejection of physical contact by the mother: aggression, avoidance, and conflict. I Am Acad Child Psychiatry 1981; 20: 292-307. doi:10.1016/s0002-7138(09)60990-0 
[40] Anisfeld E, Casper V, Nozyce M et al. Does infant carrying promote attachment? An experimental study of the effects of increased physical contact on the development of attachment. Child Dev 1990; 61: 16171627. doi:10.1111/j.1467-8624.1990.tb02888.x

[41] Balikci A, Ilbay G, Ates N. Neonatal Tactile Stimulations Affect Genetic Generalized Epilepsy and Comorbid Depression-Like Behaviors. Front Behav Neurosci 2020; 14: 132. doi:10.3389/fnbeh.2020.00132

[42] Cortés-García L, Takkouche B, Rodriguez-Cano R et al. Mediational mechanisms involved in the relation between attachment insecurity and depression: A meta-analysis. J Affect Disord 2020; 277: 706-726. doi:10.1016/j.jad.2020.08.082

[43] Macdonald JA, Greenwood C], Letcher P et al. Parent and Peer Attachments in Adolescence and Paternal Postpartum Mental Health: Findings From the ATP Generation 3 Study. Front Psychol 2021; 12: 672174. doi:10.3389/fpsyg.2021.672174

[44] Verhees, Martine W F T, Finet C, Vandesande S, et al. Attachment and the Development of Depressive Symptoms in Adolescence: The Role of Regulating Positive and Negative Affect. J Youth Adolesc 2021. doi:10.1007/s10964-021-01426-y

[45] Williams LR, Turner PR. Infant carrying as a tool to promote secure attachments in young mothers: Comparing intervention and control infants during the still-face paradigm. Infant Behav Dev 2020; 58 : 101413. doi:10.1016/j.infbeh.2019.101413

[46] Norholt H. Delivering Clinically on Our Knowledge of Oxytocin and Sensory Stimulation: The Potenzial of Infant Carrying in Primary Prevention. Front Psychol 2021; 11: 590051. doi:10.3389/fpsyg.2020.590051

[47] Varlamov AA, Portnova GV, McGlone F. The C-Tactile System and the Neurobiological Mechanisms of "Affective" Tactile Perception: The History of Discoveries and the Current State of Research. Neurosci Behav Physi 2020; 50: 418-427. doi:10.1007/s11055-020-00916-z

[48] Björnsdotter M, Morrison I, Olausson H. Feeling good: on the role of $C$ fiber mediated touch in interoception. Exp Brain Res 2010; 207: 149155. doi:10.1007/s00221-010-2408-y

[49] Gordon I, Voos AC, Bennett RH et al. Brain mechanisms for processing affective touch. Hum Brain Mapp 2013; 34: 914-922. doi:10.1002/ hbm. 21480

[50] Cascio C], Moore D, McGlone F. Social touch and human development. Dev Cogn Neurosci 2019; 35: 5-11. doi:10.1016/j.dcn.2018.04.009

[51] Cuijpers P, van Straten A, Bohlmeijer E et al. The effects of psychotherapy for adult depression are overestimated: a meta-analysis of study quality and effect size. Psychol Med 2010; 40: 211-223. doi:10.1017| S0033291709006114

[52] Kirsch I, Deacon B], Huedo-Medina TB et al. Initial severity and antidepressant benefits: a meta-analysis of data submitted to the Food and Drug Administration. PLoS Med 2008; 5: e45. doi:10.1371/ journal.pmed.0050045

[53] Hengartner MP, Angst J, Rössler W. Antidepressant Use Prospectively Relates to a Poorer Long-Term Outcome of Depression: Results from a Prospective Community Cohort Study over 30 Years. Psychother Psychosom 2018; 87: 181-183. doi:10.1159/000488802

[54] Weinmann S. Die Vermessung der Psychiatrie. Täuschung und Selbsttäuschung eines Fachgebiets. Köln: Psychiatrie Verlag; 2019

[55] van Dam NT, van Vugt MK, Vago DR et al. Mind the Hype: A Critical Evaluation and Prescriptive Agenda for Research on Mindfulness and Meditation. Perspect Psychol Sci 2018; 13: 36-61. doi:10.1177/ 1745691617709589

[56] Paykel ES, Ramana R, Cooper Z et al. Residual symptoms after partial remission: an important outcome in depression. Psychol Med 1995; 25 : 1171-1180. doi:10.1017/s0033291700033146

[57] Lyons N, Strasser A, Beitz B et al. Bodily Maps of Emotion in Major Depressive Disorder. Cognitive Therapy and Research 2020;; Online ahead of print. doi:10.1007/s10608-020-10195-0
[58] Simmons AN, Arce E, Lovero KL et al. Subchronic SSRI administration reduces insula response during affective anticipation in healthy volunteers. Int J Neuropsychopharmacol 2009; 12: 1009-1020. doi:10.1017| S1461145709990149

[59] Schmitz H. Phänomenologie der Leiblichkeit. In: Petzold H, Hrsg. Leiblichkeit. Philosophische, gesellschaftliche und therapeutische Perspektiven. Bd. 25 Paderborn: Junfermann; 1985: 71-106

[60] Fuchs T. Depression und Manie als Zeitkrankheiten. In: Fuchs T, Berger M, Hrsg. Affektive Störungen. Klinik - Therapie - Perspektiven. Klett-Cotta: Stuttgart; 2013: 171-186

[61] Rosa H. Beschleunigung und Entfremdung. Entwurf einer kritischen Theorie spätmoderner Zeitlichkeit. 6. Aufl. Berlin: Suhrkamp; 2018

[62] Cornaglia C. Körperliche Symptome bei affektiven Störungen. In: Fuchs T, Berger M, Hrsg. Affektive Störungen. Klinik - Therapie Perspektiven. Stuttgart: Klett-Cotta; 2013: 85-94

[63] Pohl H. Unerklärliche Beschwerden? Chronische Schmerzen und andere Leiden körpertherapeutisch verstehen und behandeln. München: Knaur; 2010

[64] Sloterdijk P. Sphären. Eine Trilogie. Band 1. Frankfurt am Main: Suhrkamp; 1998

[65] Röhricht F, Beyer W, Priebe S. Disturbances of Body-Experience in Acute Anxiety and Depressive Disorders - Neuroticism or Somatization? Psychother Psychosom Med Psychol 2002; 52: 205-213. doi:10.1055/s-200228524

[66] Müller-Oerlinghausen B, Berg C, Droll W. Die Slow Stroke®Massage ein körpertherapeutischer Ansatz bei Depression und anderen psychiatrischen Störungen. In: Wollschläger M, Hrsg. Hirn, Herz, Seele, Schmerz. Psychotherapie zwischen Neurowissenschaften und Geisteswissenschaften. Tübingen: Dgvt-Verl; 2008: 231-246

[67] Lyons N, Michaelsen MM, Graser ] et al. Bodily Experience in Depression: Using Focusing as a New Interview Technique. Psychopathology 2021; 54: 150-158. doi:10.1159/000514128

[68] Berg C, Müller-Oerlinghausen B. Slow Stroke Massage. In: Reichert B, Hrsg. Massage-Therapie. Stuttgart, New York: Georg Thieme Verlag; 2015: 328-337

[69] Müller-Oerlinghausen B. Psychische Effekte und ihre klinische Wirksamkeit. In: Reichert B, Hrsg. Massage-Therapie. Stuttgart, New York: Georg Thieme Verlag; 2015: 41-46

[70] Arnold MM, Müller-Oerlinghausen B, Hemrich N et al. Effects of Psychoactive Massage in Outpatients with Depressive Disorders: A Randomized Controlled Mixed-Methods Study. Brain Sci 2020; 10: 676. doi:10.3390/ brainsci10100676

[71] Kiebgis GM, Eggart M, Thormählen C et al. Psychophysische Kurzzeitund Langzeiteffekte einer einmaligen Massageanwendung - Ergebnisse einer naturalistischen Studie. Phys Med Rehab Kuror 2018; 28: 120-128. doi:10.1055/s-0043-124471

[72] Emrich HM. Depression und Berührung. Vortrag auf dem Symposion „Optimierung der antidepressiven Therapie“. Berlin, 2001

[73] Maurer-Groeli YA. Die Haut als Medium in der Therapie depressiv und schizophren Kranker. Psychosomatische Medizin 1975; 6: 67-78

[74] Moyer CA, Rounds J, Hannum JW. A meta-analysis of massage therapy research. Psychol Bull 2004; 130: 3-18. doi:10.1037/0033-2909.130.1.3

[75] Baumgart S, Müller-Oerlinghausen B, Schendera CF. Wirksamkeit der Massagetherapie bei Depression und Angsterkrankungen sowie bei Depressivität und Angst als Komorbidität - Eine systematische Übersicht kontrollierter Studien. Phys Med Rehab Kuror 2011; 21: 167-182. doi: $10.1055 / \mathrm{s}-0031-1279760$

[76] Müller-Oerlinghausen B, Berg C, Scherer P et al. Effects of slow-stroke massage as complementary treatment of depressed hospitalized patients. Results of a controlled study (SeSeTra). Dtsch Med Wochenschr 2004; 129 : 1363-1368. doi:10.1055/s-2004-826874 
[77] Baumgart SB-E, Baumbach-Kraft A, Lorenz ]. Effect of Psycho-Regulatory Massage Therapy on Pain and Depression in Women with Chronic and/or Somatoform Back Pain: A Randomized Controlled Trial. Brain Sci 2020; 10: 721. doi:10.3390/brainsci10100721

[78] Müller-Oerlinghausen B, Eggart M. Touch Research-Quo Vadis? A Plea for High-Quality Clinical Trials. Brain Sci 2021; 11: 25. doi:10.3390/ brainsci11010025

[79] Möller JU. Psychologische Aspekte der Massage. Lymphologie 1994; 18 : 16-19

[80] Cassileth BR, Vickers AJ. Massage therapy for symptom control: outcome study at a major cancer center. J Pain Symptom Manage 2004; 28: 244249. doi:10.1016/j.jpainsymman.2003.12.016

[81] Listing M, Reisshauer A, Krohn M et al. Massage therapy reduces physical discomfort and improves mood disturbances in women with breast cancer. Psychooncology 2009; 18: 1290-1299. doi:10.1002/pon.1508

[82] Uvnäs Moberg K. Oxytocin may mediate the benefits of positive social interaction and emotions. Psychoneuroendocrinology 1998; 23: 819-835. doi:10.1016/s0306-4530(98)00056-0

[83] Uvnäs Moberg K, Handlin L, Petersson M. Self-soothing behaviors with particular reference to oxytocin release induced by non-noxious sensory stimulation. Front Psychol 2015; 5: 1529. doi:10.3389/ fpsyg.2014.01529

[84] Ludwig M, Leng G. Dendritic peptide release and peptide-dependent behaviours. Nat Rev Neurosci 2006; 7: 126-136. doi:10.1038/nrn1845

[85] Sofroniew MV. Vasopressin and oxytocin in the mammalian brain and spinal cord. Trends Neurosci 1983; 6: 467-472. doi:10.1016/01662236(83)90221-7

[86] Knobloch HS, Charlet A, Hoffmann LC et al. Evoked axonal oxytocin release in the central amygdala attenuates fear response. Neuron 2012; 73: 553-566. doi:10.1016/j.neuron.2011.11.030

[87] Stoop R, Hegoburu C, van den Burg E. New opportunities in vasopressin and oxytocin research: a perspective from the amygdala. Annu Rev Neurosci 2015; 38: 369-388. doi:10.1146/annurev-neuro-071714033904

[88] Uvnäs Moberg K, Petersson M. Oxytocin, ein Vermittler von Antistress, Wohlbefinden, sozialer Interaktion, Wachstum und Heilung. Z Psychosom Med Psychother 2005; 51: 57-80. doi:10.13109/zptm.2005.51.1.57

[89] Uvnäs Moberg K, Ekström-Bergström A, Berg M et al. Maternal plasma levels of oxytocin during physiological childbirth - a systematic review with implications for uterine contractions and central actions of oxytocin. BMC Pregnancy Childbirth 2019; 19: 285. doi:10.1186/s12884-019-2365-9

[90] Uvnäs Moberg K, Ekström-Bergström A, Buckley S et al. Maternal plasma levels of oxytocin during breastfeeding-A systematic review. PLOS ONE 2020; 15: e0235806. doi:10.1371/journal.pone.0235806

[91] Stock S, Uvnäs Moberg K. Increased plasma levels of oxytocin in response to afferent electrical stimulation of the sciatic and vagal nerves and in response to touch and pinch in anaesthetized rats. Acta Physiol Scand 1988; 132: 29-34. doi:10.1111/j.1748-1716.1988.tb08294.x
[92] Uvnäs Moberg K, Handlin L, Petersson M. Neuroendocrine mechanisms involved in the physiological effects caused by skin-to-skin contact With a particular focus on the oxytocinergic system. Infant Behav Dev 2020; 61: 101482. doi:10.1016/j.infbeh.2020.101482

[93] Sofroniew MV. Projections from vasopressin, oxytocin, and neurophysin neurons to neural targets in the rat and human. J Histochem Cytochem 1980; 28: 475-478. doi:10.1177/28.5.7381192

[94] Buis RM. Vasopressin and oxytocin - their role in neurotransmission. Pharmacol Ther 1983; 22: 127-141

[95] Takahashi T. Sensory Stimulation of Oxytocin Release Is Associated With Stress Management and Maternal Care. Front Psychol 2020; 11: 588068. doi:10.3389/fpsyg.2020.588068

[96] Field T. Massage therapy research review. Complement Ther Clin Pract 2016; 24: 19-31. doi:10.1016/j.ctcp.2016.04.005

[97] Craig AD. How do you feel? Interoception: the sense of the physiological condition of the body. Nat Rev Neurosci 2002; 3: 655-666. doi:10.1038/ nrn894

[98] Craig AD. How do you feel - now? The anterior insula and human awareness. Nat Rev Neurosci 2009; 10: 59-70. doi:10.1038/nrn2555

[99] James W. What is an emotion? Mind 1884; 9: 188-205

[100] Eggart M, Lange A, Binser M] et al. Major depressive disorder is associated with impaired interoceptive accuracy: a systematic review. Brain Sci 2019; 9: 131. doi:10.3390/brainsci9060131

[101] Eggart M, Valdés-Stauber J. Can changes in multidimensional self-reported interoception be considered as outcome predictors in severely depressed patients? A moderation and mediation analysis. Advance online publication. J Psychosom Res 2021; 141. doi:10.1016/j.jpsychores.2020.110331

[102] Eggart M, Todd J, Valdés-Stauber J. Validation of the Multidimensional Assessment of Interoceptive Awareness (MAIA-2) questionnaire in hospitalized patients with major depressive disorder. PLoS ONE 2021; 16: e0253913. doi:10.1371/journal.pone.0253913

[103] Eggart M, Queri S, Müller-Oerlinghausen B. Are the antidepressive effects of massage therapy mediated by restoration of impaired interoceptive functioning? A novel hypothetical mechanism. Med Hypotheses 2019; 128: 28-32. doi:10.1016/j.mehy.2019.05.004

[104] Cerritelli F, Chiacchiaretta P, Gambi F et al. Effect of manual approaches with osteopathic modality on brain correlates of interoception: an fMRI study. Sci Rep 2020; 10: 3214. doi:10.1038/s41598-020-60253-6

[105] Edwards DJ, Young H, Cutis A et al. The Immediate Effect of Therapeutic Touch and Deep Touch Pressure on Range of Motion, Interoceptive Accuracy and Heart Rate Variability: A Randomized Controlled Trial With Moderation Analysis. Front Integr Neurosci 2018; 12: 41. doi:10.3389/ fnint.2018.00041

[106] Koch C, Wilhelm M, Salzmann S et al. A meta-analysis of heart rate variability in major depression. Psychol Med 2019; 49: 1948-1957. doi:10.1017/S0033291719001351

[107] Margenfeld F, Klocke C, Joos S. Manual massage for persons living with dementia: A systematic review and meta-analysis. Int J Nurs Stud 2019; 96: 132-142. doi:10.1016/j.jinurstu.2018.12.012 